\title{
THE GENERALIZED AUSLANDER-REITEN DUALITY ON AN EXACT CATEGORY
}

\author{
PENGJIE JIAO
}

\begin{abstract}
We introduce a notion of generalized Auslander-Reiten duality on a Hom-finite Krull-Schmidt exact category $\mathcal{C}$. This duality induces the generalized Auslander-Reiten translation functors $\tau$ and $\tau^{-}$. They are mutually quasi-inverse equivalences between the stable categories of two full subcategories $\mathcal{C}_{r}$ and $\mathcal{C}_{l}$ of $\mathcal{C}$. A non-projective indecomposable object lies in the domain of $\tau$ if and only if it appears as the third term of an almost split conflation; dually, a non-injective indecomposable object lies in the domain of $\tau^{-}$if and only if it appears as the first term of an almost split conflation. We study the generalized Auslander-Reiten duality on the category of finitely presented representations of locally finite interval-finite quivers.
\end{abstract}

\section{INTRODUCTION}

Throughout, $k$ denotes a commutative artinian ring. We consider $k$-linear skeletally small exact categories.

Recall that an abelian category $\mathcal{A}$ is said to have enough almost split sequences provided that each non-projective indecomposable object appears as the third term of an almost split sequence, and that each non-injective indecomposable object appears as the first term of an almost split sequence.

H. Lenzing and R. Zuazua proved that an Ext-finite abelian category $\mathcal{A}$ has enough almost split sequences if and only if it has Auslander-Reiten duality; see 8 , Theorem 1.1]. S. Liu, P. Ng and C. Paquette 9 investigated almost split sequences in an exact category, and proved the local version of [8, Theorem 1.1] under weaker hypotheses; see [9, Theorem 3.6]. One can observe that the notion of AuslanderReiten duality applies to exact categories naturally.

Inspired by [5], we introduce the notion of generalized Auslander-Reiten duality for a Hom-finite Krull-Schmidt exact category $\mathcal{C}$. This duality induces the generalized Auslander-Reiten translation functors $\tau$ and $\tau^{-}$, which are defined on the stable categories of two full subcategories $\mathcal{C}_{r}$ and $\mathcal{C}_{l}$ of $\mathcal{C}$. Then $\mathcal{C}$ has AuslanderReiten duality in the sense of $\left[8\right.$ if and only if $\mathcal{C}_{l}=\mathcal{C}=\mathcal{C}_{r}$. In general, $\mathcal{C}_{r}$ and $\mathcal{C}_{l}$ are not equal to $\mathcal{C}$.

We prove that a non-projective indecomposable object lies in $\mathcal{C}_{r}$ if and only if it appears as the third term of an almost split conflation, and that a non-injective indecomposable object lies in $\mathcal{C}_{l}$ if and only if it appears as the first term of an almost

Date: July 12, 2018.

2010 Mathematics Subject Classification. 16G70, 16G20, 18E10.

Key words and phrases. almost split conflation, Auslander-Reiten duality, stable category, representations of infinite quivers. 
split conflation; see Proposition 2.4. We prove that the generalized AuslanderReiten translation functors $\tau$ and $\tau^{-}$are mutually quasi-inverse equivalences between the projectively stable category of $\mathcal{C}_{r}$ and the injectively stable category of $\mathcal{C}_{l}$; see Proposition 3.4 .

In Section 4, we describe the full subcategories $\mathcal{C}_{r}$ and $\mathcal{C}_{l}$, and the generalized Auslander-Reiten translation functors $\tau$ and $\tau^{-}$in the case that $\mathcal{C}$ is the category of finitely presented representations of a locally finite interval-finite quiver. This description depends on the results of [3].

\section{TWO FULL SUBCATEGORIES}

Let $k$ be a commutative artinian ring and $\check{k}$ be the minimal injective cogenerator. Denote by $k$-mod the category of finitely generated $k$-modules and by $D=\operatorname{Hom}_{k}(-, \check{k})$ the Matlis duality.

Let $\mathcal{C}$ be a $k$-linear exact category. Recall that an exact category is an additive category $\mathcal{C}$ together with a collection $\mathcal{E}$ of kernel-cokernel pairs $(i, d)$ which satisfies the axioms in [7, Appendix A]; compare [11, Section 2]. Here, a kernel-cokernel pair $(i, d)$ means a sequence of morphisms $X \stackrel{i}{\rightarrow} Y \stackrel{d}{\rightarrow} Z$ satisfying that $i$ is the kernel of $d$ and $d$ is the cokernel of $i$. A kernel-cokernel pair $(i, d)$ in $\mathcal{E}$ is called a conflation, while $i$ is called an inflation and $d$ is called a deflation. For two objects $X$ and $Y$ in $\mathcal{C}$, we denote by $\operatorname{Ext}_{\mathcal{E}}^{1}(X, Y)$ the set of equivalence classes of conflations $Y \rightarrow E \rightarrow X$. Given a conflation $\mu: Y \rightarrow E \rightarrow X$, for each $f: Z \rightarrow X$, we denote by $\mu . f=\operatorname{Ext}_{\mathcal{E}}^{1}(f, Y)(\mu)$ the conflation obtained by the pullback of $\mu$ along $f$. Dually, for each $g: Y \rightarrow Z$, we denote by $g \cdot \mu=\operatorname{Ext}_{\mathcal{E}}^{1}(X, g)(\mu)$ the conflation obtained by the pushout of $\mu$ along $g$.

Recall from [8, Section 2] that a morphism $f: X \rightarrow Y$ is projectively triv$i a l$ provided that for each object $Z$, the induced map $\operatorname{Ext}_{\mathcal{E}}^{1}(f, Z): \operatorname{Ext}_{\mathcal{E}}^{1}(Y, Z) \rightarrow$ $\operatorname{Ext}_{\mathcal{E}}^{1}(X, Z)$ is zero. We observe that $f$ is projectively trivial if and only if $f$ factors through each deflation $E \rightarrow Y$ ending at $Y$. Dually, a morphism $f$ is injectively trivial provided that for each object $Z$, the induced map $\operatorname{Ext}_{\mathcal{E}}^{1}(Z, f): \operatorname{Ext}_{\mathcal{E}}^{1}(Z, X) \rightarrow$ $\operatorname{Ext}_{\mathcal{E}}^{1}(Z, Y)$ is zero.

Given two objects $X$ and $Y$, we denote by $\mathcal{P}(X, Y)$ the set of projectively trivial morphisms from $X$ to $Y$. Then $\mathcal{P}$ forms an ideal of $\mathcal{C}$. The projectively stable category $\underline{\mathcal{C}}$ of $\mathcal{C}$ is the factor category $\mathcal{C} / \mathcal{P}$. Given a morphism $f: X \rightarrow Y$, we denote by $\underline{f}$ its image in $\underline{\mathcal{C}}$. We denote by $\underline{\operatorname{Hom}}_{\mathcal{C}}(X, Y)=\operatorname{Hom}_{\mathcal{C}}(X, Y) / \mathcal{P}(X, Y)$ the set of morphisms in $\underline{\mathcal{C}}$. Given an object $Z$, we mention that $\operatorname{Ext}_{\mathcal{E}}^{1}(-, Z)$ is a contravariant functor from $\underline{\mathcal{C}}$ to the category of $k$-modules. We observe that an object $P$ becomes zero in $\underline{\mathcal{C}}$ if and only if $P$ is projective in $\mathcal{C}$.

Dually, we denote by $\mathcal{I}(X, Y)$ the set of injectively trivial morphisms from $X$ to $Y$. The injectively stable category $\overline{\mathcal{C}}$ of $\mathcal{C}$ is the factor category $\mathcal{C} / \mathcal{I}$. Given a morphism $f: X \rightarrow Y$, we denote by $\bar{f}$ its image in $\overline{\mathcal{C}}$. We denote by $\overline{\operatorname{Hom}}_{\mathcal{C}}(X, Y)=$ $\operatorname{Hom}_{\mathcal{C}}(X, Y) / \mathcal{I}(X, Y)$ the set of morphisms in $\overline{\mathcal{C}}$. We mention that $\operatorname{Ext}_{\mathcal{E}}^{1}(Z,-)$ is a functor from $\overline{\mathcal{C}}$ to the category of $k$-modules. We observe that an object $I$ becomes zero in $\overline{\mathcal{C}}$ if and only if $I$ is injective in $\mathcal{C}$.

Recall that a morphism $v: E \rightarrow Y$ is right almost split if it is not a retraction and each $f: Z \rightarrow Y$ which is not a retraction factors through $v$. Dually, a morphism $u: X \rightarrow E$ is left almost split if it is not a section and each $f: X \rightarrow Z$ which is not a section factors through $u$. A conflation $\delta: X \stackrel{u}{\rightarrow} E \stackrel{v}{\rightarrow} Y$ is an almost 
split conflation if $u$ is left almost split and $v$ is right almost split. Recall that an object whose endomorphism algebra is local is called strongly indecomposable. We mention that given an almost split conflation $X \rightarrow E \rightarrow Y$, the objects $X$ and $Y$ are strongly indecomposable; see [1, Proposition II.4.4]. We refer to [2, Chapter V] for the general properties of almost split conflations.

We observe the fact that for each non-split conflation $\mu: X \rightarrow Y \rightarrow Z$, there exists some $\gamma \in D \operatorname{Ext}_{\mathcal{E}}^{1}(Z, X)$ such that $\gamma(\mu) \neq 0$. The following lemma is essentially due to [8, Proposition 3.1]; compare [6. Theorem 9.3 and Corollary 9.4] and [9. Proposition 3.1].

Lemma 2.1. Let $\delta: X \rightarrow E \rightarrow Y$ be an almost split conflation and let $\gamma \in$ $D \operatorname{Ext}_{\mathcal{E}}^{1}(Y, X)$ with $\gamma(\delta) \neq 0$. Then we have the following statements.

(1) For each $M$, we have a non-degenerate $k$-bilinear map

$$
\langle-,-\rangle_{M}: \overline{\operatorname{Hom}}_{\mathcal{C}}(M, X) \times \operatorname{Ext}_{\mathcal{E}}^{1}(Y, M) \longrightarrow \check{k}, \quad(\bar{f}, \mu) \mapsto \gamma(f \cdot \mu) .
$$

If moreover $\overline{\operatorname{Hom}}_{\mathcal{C}}(M, X) \in k$-mod for each $M$, then the induced map

$$
\phi_{Y, M}: \overline{\operatorname{Hom}}_{\mathcal{C}}(M, X) \longrightarrow D \operatorname{Ext}_{\mathcal{E}}^{1}(Y, M), \quad \bar{f} \mapsto\langle\bar{f},-\rangle_{M},
$$

is an isomorphism and natural in $M$ with $\gamma=\phi_{Y, X}\left(\overline{\operatorname{Id}_{X}}\right)$.

(2) For each $M$, we have a non-degenerate $k$-bilinear map

$$
\langle-,-\rangle_{M}: \operatorname{Ext}_{\mathcal{E}}^{1}(M, X) \times \underline{\operatorname{Hom}}_{\mathcal{C}}(Y, M) \longrightarrow \check{k}, \quad(\mu, \underline{g}) \mapsto \gamma(\mu . g) .
$$

If moreover $\underline{\operatorname{Hom}}_{\mathcal{C}}(Y, M) \in k$-mod for each $M$, then the induced map

$$
\psi_{X, M}: \underline{\operatorname{Hom}}_{\mathcal{C}}(Y, M) \longrightarrow D \operatorname{Ext}_{\mathcal{E}}^{1}(M, X), \quad \underline{g} \mapsto\langle-, \underline{g}\rangle_{M},
$$

is an isomorphism and natural in $M$ with $\gamma=\psi_{X, Y}\left(\underline{\operatorname{Id}_{Y}}\right)$.

Proof. (1) It is sufficient to show that the $k$-bilinear map $\langle-,-\rangle_{M}$ is non-degenerate for each $M$. The naturalness of $\phi_{Y}: \overline{\operatorname{Hom}}_{\mathcal{C}}(-, X) \rightarrow D \operatorname{Ext}_{\mathcal{E}}^{1}(Y,-)$ follows from a direct verification.

On the one hand, assume that $\mu: M \rightarrow E^{\prime} \rightarrow Y$ is a non-split conflation. Since $\delta$ is almost split, we obtain the following commutative diagram

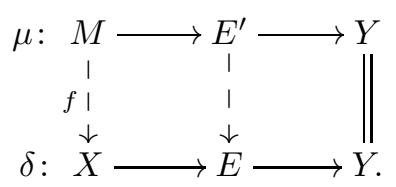

Here, the left square is a pushout diagram. Hence $f . \mu=\delta$ and $\langle\bar{f}, \mu\rangle_{M}=\gamma(\delta) \neq 0$.

On the other hand, let $\bar{f}: M \rightarrow X$ be a nonzero morphism in $\overline{\mathcal{C}}$. We have that $f$ is not injectively trivial in $\mathcal{C}$. Hence there exists some object $N$ and some $\mu \in \operatorname{Ext}_{\mathcal{E}}^{1}(N, M)$ such that $f . \mu=\operatorname{Ext}_{\mathcal{E}}^{1}(N, f)(\mu)$ is non-split. Since $\delta$ is almost split, it can be obtained by a pullback of $f . \mu$ along some $h: Y \rightarrow N$.

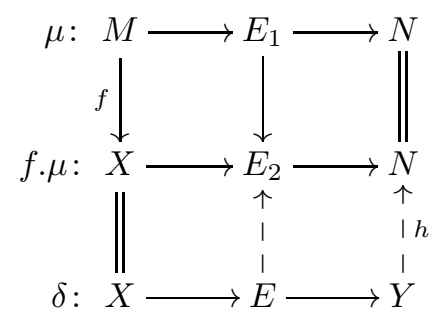


Hence we have $\delta=(f . \mu) \cdot h=f .(\mu . h)$ and then $\langle\bar{f}, \mu . h\rangle_{M}=\gamma(\delta) \neq 0$.

(2) The proof is similar.

The following lemma is a slight modification of [8, Proposition 4.1]; compare [9, Theorem 3.6].

Lemma 2.2. Let $Y$ be a strongly indecomposable object in $\mathcal{C}$.

(1) Assume $D \operatorname{Ext}_{\mathcal{E}}^{1}(Y,-) \simeq \overline{\operatorname{Hom}}_{\mathcal{C}}\left(-, Y^{\prime}\right)$ for some $Y^{\prime}$. If $\overline{\operatorname{Hom}}_{\mathcal{C}}\left(Z, Y^{\prime}\right) \in$ $k$-mod for each $Z$, and $Y^{\prime}$ has a non-injective strongly indecomposable direct summand, then there exists an almost split conflation ending at $Y$.

(2) Assume $D \operatorname{Ext}_{\mathcal{E}}^{1}(-, Y) \simeq \underline{\operatorname{Hom}}_{\mathcal{C}}\left(Y^{\prime},-\right)$ for some $Y^{\prime}$. If $\underline{\operatorname{Hom}}_{\mathcal{C}}\left(Y^{\prime}, Z\right) \in$ $k$-mod for each $Z$, and $Y^{\prime}$ has a non-projective strongly indecomposable direct summand, then there exists an almost split conflation starting at $Y$.

Proof. (1) Let $\phi: \overline{\operatorname{Hom}}_{\mathcal{C}}\left(-, Y^{\prime}\right) \rightarrow D \operatorname{Ext}_{\mathcal{E}}^{1}(Y,-)$ be an isomorphism of functors. Set $\gamma=\phi_{Y^{\prime}}\left(\overline{\operatorname{Id}_{Y^{\prime}}}\right)$. By the naturalness of $\phi$, for each object $M$ and each morphism $f: M \rightarrow Y^{\prime}$, we have

$$
\phi_{M}(\bar{f})=D \operatorname{Ext}_{\mathcal{E}}^{1}(Y, f)(\gamma)=\gamma \circ \operatorname{Ext}_{\mathcal{E}}^{1}(Y, f) .
$$

It follows that $\phi_{M}(\bar{f})(\mu)=\gamma(f . \mu)$ for each $\mu \in \operatorname{Ext}_{\mathcal{E}}^{1}(Y, M)$.

Let $X$ be a non-injective strongly indecomposable direct summand of $Y^{\prime}$. Then the isomorphism $\phi_{X}$ induces a non-degenerate $k$-bilinear map

$$
\langle-,-\rangle_{X}: \overline{\operatorname{Hom}}_{\mathcal{C}}\left(X, Y^{\prime}\right) \times \operatorname{Ext}_{\mathcal{E}}^{1}(Y, X) \longrightarrow \check{k}, \quad(\bar{f}, \mu) \mapsto \gamma(f . \mu) .
$$

Let $H \subseteq \operatorname{Hom}_{\mathcal{C}}\left(X, Y^{\prime}\right)$ be the subset formed by non-sections. Observe that $H$ is a $k$-submodule since $X$ is strongly indecomposable. We have that $\mathcal{I}\left(X, Y^{\prime}\right) \subseteq H$ since $X$ is non-injective. Hence $H / \mathcal{I}\left(X, Y^{\prime}\right)$ is a proper $k$-submodule of $\overline{\operatorname{Hom}}_{\mathcal{C}}\left(X, Y^{\prime}\right)$. Then there exists some nonzero $\alpha \in D \overline{\operatorname{Hom}}_{\mathcal{C}}\left(X, Y^{\prime}\right)$ such that $\alpha(\bar{f})=0$ for each $f \in H$. By the non-degenerate bilinear form $\langle-,-\rangle_{X}$, there exists some non-split conflation $\delta: X \stackrel{u}{\rightarrow} E \rightarrow Y$ such that $\alpha=\langle-, \delta\rangle_{X}$. Then we have $\langle\bar{f}, \delta\rangle_{X}=0$ for each $f \in H$.

We claim that $u$ is left almost split. Indeed, we observe that $u$ is not a section. Assume that $h: X \rightarrow M$ is not a section. Then for each $g: M \rightarrow Y^{\prime}$, the morphism $g \circ h$ is not a section and thus lies in $H$. Hence we have $\langle\overline{g \circ h}, \delta\rangle_{X}=0$. Consider the non-degenerate $k$-bilinear map

$$
\langle-,-\rangle_{M}: \overline{\operatorname{Hom}}_{\mathcal{C}}\left(M, Y^{\prime}\right) \times \operatorname{Ext}_{\mathcal{E}}^{1}(Y, M) \longrightarrow \check{k}, \quad(\bar{f}, \mu) \mapsto \gamma(f \cdot \mu),
$$

induced by $\phi_{M}$. For each $g: M \rightarrow Y^{\prime}$, we have

$$
\langle\bar{g}, h . \delta\rangle_{M}=\gamma(g .(h . \delta))=\langle\overline{g \circ h}, \delta\rangle_{X}=0 .
$$

This implies that the conflation $h . \delta$ splits. In other words, the morphism $h$ factors through $u$. Therefore the morphism $u$ is left almost split. It follows from [1, Proposition II.4.4] that $\delta$ is an almost split conflation since $\operatorname{End}_{\mathcal{C}}(Y)$ is local.

(2) The proof is similar.

From now on, we assume that the $k$-linear exact category is Hom-finite and KrullSchmidt. Here, the Hom-finiteness means that all the Hom $k$-modules are finitely generated. In this case all indecomposable objects are strongly indecomposable. Moreover, the stable categories $\underline{\mathcal{C}}$ and $\overline{\mathcal{C}}$ are Krull-Schmidt and thus have split idempotents. 
We introduce two full subcategories of $\mathcal{C}$ as follows

$$
\mathcal{C}_{r}=\left\{X \in \mathcal{C} \mid \text { the functor } D \operatorname{Ext}_{\mathcal{E}}^{1}(X,-): \overline{\mathcal{C}} \rightarrow k \text {-mod is representable }\right\}
$$

and

$$
\mathcal{C}_{l}=\left\{X \in \mathcal{C} \mid \text { the functor } D \operatorname{Ext}_{\mathcal{E}}^{1}(-, X): \underline{\mathcal{C}} \rightarrow k \text {-mod is representable }\right\} .
$$

Here, both $\mathcal{C}_{r}$ and $\mathcal{C}_{l}$ are additive subcategories which are closed under direct summands. Indeed, it follows from Yoneda's lemma and the fact that $\underline{\mathcal{C}}$ and $\overline{\mathcal{C}}$ have split idempotents.

Lemma 2.3. Let $X$ and $Y$ be two objects in $\mathcal{C}$.

(1) Assume that $X \in \mathcal{C}_{r}$ and $X \simeq Y$ in $\underline{\mathcal{C}}$. Then the object $Y$ lies in $\mathcal{C}_{r}$.

(2) Assume that $X \in \mathcal{C}_{l}$ and $X \simeq Y$ in $\overline{\mathcal{C}}$. Then the object $Y$ lies in $\mathcal{C}_{l}$.

Proof. (1) We observe that $D \operatorname{Ext}_{\mathcal{E}}^{1}(X,-) \simeq D \operatorname{Ext}_{\mathcal{E}}^{1}(Y,-)$ as functors. Then the result follows. The proof of $(2)$ is similar.

As a consequence of Lemmas 2.1 and 2.2, we have the following description of indecomposable objects in $\mathcal{C}_{r}$ and $\mathcal{C}_{l}$.

Proposition 2.4. Let $Y$ be an indecomposable object in $\mathcal{C}$.

(1) Assume that $Y$ is non-projective. Then $Y \in \mathcal{C}_{r}$ if and only if there exists an almost split conflation ending at $Y$.

(2) Assume that $Y$ is non-injective. Then $Y \in \mathcal{C}_{l}$ if and only if there exists an almost split conflation starting at $Y$.

\section{An equivalence between Stable subCategories}

Let $\mathcal{C}$ be a Hom-finite Krull-Schmidt exact category. For each object $Y$ in $\mathcal{C}_{r}$, we fix some isomorphism of functors

$$
\phi_{Y}: \overline{\operatorname{Hom}}_{\mathcal{C}}(-, \tau Y) \longrightarrow D \operatorname{Ext}_{\mathcal{E}}^{1}(Y,-) .
$$

Then $\tau$ gives a map from the objects of $\mathcal{C}_{r}$ to $\mathcal{C}$. Dually, for each object $X$ in $\mathcal{C}_{l}$, we fix some isomorphism of functors

$$
\psi_{X}: \underline{\operatorname{Hom}}_{\mathcal{C}}\left(\tau^{-} X,-\right) \longrightarrow D \operatorname{Ext}_{\mathcal{E}}^{1}(-, X) .
$$

Then $\tau^{-}$gives a map from the objects of $\mathcal{C}_{l}$ to $\mathcal{C}$.

Lemma 3.1. Let $X$ and $Y$ be two objects in $\mathcal{C}$.

(1) If $X, Y \in \mathcal{C}_{r}$ and $X \simeq Y$ in $\underline{\mathcal{C}}$, then we have $\tau X \simeq \tau Y$ in $\overline{\mathcal{C}}$.

(2) If $X, Y \in \mathcal{C}_{l}$ and $X \simeq Y$ in $\overline{\mathcal{C}}$, then we have $\tau^{-} X \simeq \tau^{-} Y$ in $\underline{\mathcal{C}}$.

Proof. (1) We observe that $\overline{\operatorname{Hom}}_{\mathcal{C}}(-, \tau X) \simeq \overline{\operatorname{Hom}}_{\mathcal{C}}(-, \tau Y)$, since they are both isomorphic to $D \operatorname{Ext}_{\mathcal{E}}^{1}(X,-) \simeq D \operatorname{Ext}_{\mathcal{E}}^{1}(Y,-)$. Then the result follows from Yoneda's lemma. The proof of $(2)$ is similar.

Lemma 3.2. Let $Y$ be an object in $\mathcal{C}$.

(1) If $Y \in \mathcal{C}_{r}$, then $\tau Y \in \mathcal{C}_{l}$ and $Y \simeq \tau^{-} \tau Y$ in $\underline{\mathcal{C}}$.

(2) If $Y \in \mathcal{C}_{l}$, then $\tau^{-} Y \in \mathcal{C}_{r}$ and $Y \simeq \tau \tau^{-} Y$ in $\overline{\mathcal{C}}$. 
Proof. We only prove (1). We may assume that $Y$ is indecomposable and nonprojective. By Lemma 2.2(1), there exists some almost split conflation $X \rightarrow E \rightarrow$ $Y$. Then we have $\overline{\operatorname{Hom}}_{\mathcal{C}}(-, X) \simeq D \operatorname{Ext}_{\mathcal{E}}^{1}(Y,-)$ and $\underline{\operatorname{Hom}}_{\mathcal{C}}(Y,-) \simeq D \operatorname{Ext}_{\mathcal{E}}^{1}(-, X)$ by Lemma 2.1. We then obtain $X \in \mathcal{C}_{l}$. It follows from Yoneda's lemma that $\tau Y \simeq X$ in $\overline{\mathcal{C}}$, and $\tau^{-} X \simeq Y$ in $\underline{\mathcal{C}}$. Hence we have $\tau Y \in \mathcal{C}_{l}$ by Lemma 2.3(2). Then we have $\tau^{-} \tau Y \simeq \tau^{-} X \simeq Y$ in $\underline{\mathcal{C}}$. Here, the first isomorphism follows from Lemma $3.1(2)$.

We denote by $\underline{\mathcal{C}_{r}}$ the image of $\mathcal{C}_{r}$ under the canonical functor $\mathcal{C} \rightarrow \underline{\mathcal{C}}$, and by $\overline{\mathcal{C}_{l}}$ the image of $\mathcal{C}_{l}$ under the canonical functor $\mathcal{C} \rightarrow \overline{\mathcal{C}}$. We will make $\tau$ into a functor from $\underline{\mathcal{C}}_{r}$ to $\overline{\mathcal{C}_{l}}$, and $\tau^{-}$into a functor from $\overline{\mathcal{C}_{l}}$ to $\underline{\mathcal{C}_{r}}$.

For each morphism $f: Y \rightarrow Y^{\prime}$ in $\mathcal{C}_{r}$, define the morphism $\tau(f): \tau Y \rightarrow \tau Y^{\prime}$ in $\overline{\mathcal{C}_{l}}$ such that the following diagram commutes

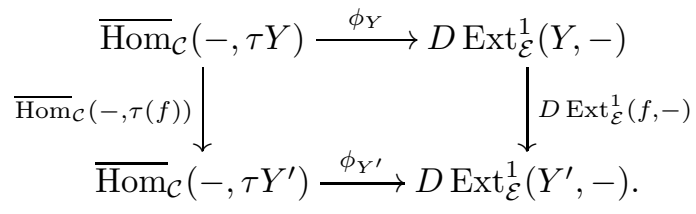

Here, the existence and uniqueness of $\tau(f)$ are guaranteed by Yoneda's lemma. Then it follows that $\tau$ is a functor from $\mathcal{C}_{r}$ to $\overline{\mathcal{C}_{l}}$. Moreover, if $f$ is projectively trivial, then $D \operatorname{Ext}_{\mathcal{E}}^{1}(f,-)=0$ and thus $\tau(f)=0$ in $\overline{\mathcal{C}_{l}}$. Hence $\tau$ induces a functor from $\underline{\mathcal{C}_{r}}$ to $\overline{\mathcal{C}_{l}}$ which we still denote by $\tau$.

Similarly, we have a functor $\tau^{-}: \overline{\mathcal{C}_{l}} \rightarrow \underline{\mathcal{C}_{r}}$. For each $\bar{g}: X \rightarrow X^{\prime}$ in $\overline{\mathcal{C}_{l}}$, the morphism $\tau^{-}(\bar{g}): \tau^{-} X \rightarrow \tau^{-} X^{\prime}$ is given by the following commutative diagram

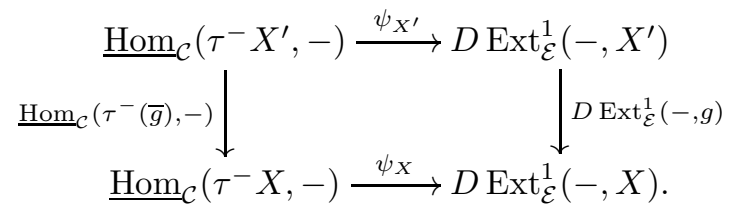

We will show that the functors $\tau$ and $\tau^{-}$are mutually quasi-inverse equivalences between $\underline{\mathcal{C}_{r}}$ and $\overline{\mathcal{C}_{l}}$.

For each object $Y \in \underline{\mathcal{C}_{r}}$, we set

$$
\underline{\epsilon_{Y}}=\left(\psi_{\tau Y, Y}^{-1} \circ \phi_{Y, \tau Y}\right)\left(\overline{\operatorname{Id}_{\tau Y}}\right): \tau^{-} \tau Y \longrightarrow Y
$$

in $\underline{\mathcal{C}_{r}}$. For each object $X \in \overline{\mathcal{C}_{l}}$, we set

$$
\overline{\eta_{X}}=\left(\phi_{\tau^{-} X, X}^{-1} \circ \psi_{X, \tau^{-} X}\right)\left(\underline{\operatorname{Id}_{\tau^{-} X}}\right): X \longrightarrow \tau \tau^{-} X
$$

in $\overline{\mathcal{C}_{l}}$.

Lemma 3.3. Let $\underline{\epsilon_{Y}}$ and $\overline{\eta_{X}}$ be as above.

(1) The morphisms $\underline{\epsilon}_{Y}$ yield a natural transformation $\underline{\epsilon}: \tau^{-} \tau \rightarrow \operatorname{Id}_{\underline{\mathcal{C}_{r}}}$, and for each morphism $\underline{f}: Y \rightarrow Y^{\prime}$ in $\underline{\mathcal{C}_{r}}$ we have

$$
\tau(\underline{f})=\left(\phi_{Y^{\prime}, \tau Y}^{-1} \circ \psi_{\tau Y, Y^{\prime}}\right)\left(\underline{f} \circ \underline{\epsilon_{Y}}\right) .
$$

(2) The morphisms $\overline{\eta_{X}}$ yield a natural transformation $\bar{\eta}: \operatorname{Id}_{\overline{\mathcal{C}_{l}}} \rightarrow \tau \tau^{-}$, and for each morphism $\bar{g}: X \rightarrow X^{\prime}$ in $\overline{\mathcal{C}_{l}}$ we have

$$
\tau^{-}(\bar{g})=\left(\psi_{X, \tau^{-} X^{\prime}}^{-1} \circ \phi_{\tau^{-} X^{\prime}, X}\right)\left(\overline{\eta_{X^{\prime}}} \circ \bar{g}\right) .
$$


Proof. (1) For each $\underline{f}: Y \rightarrow Y^{\prime}$ in $\underline{\mathcal{C}_{r}}$, we have the following commutative diagram

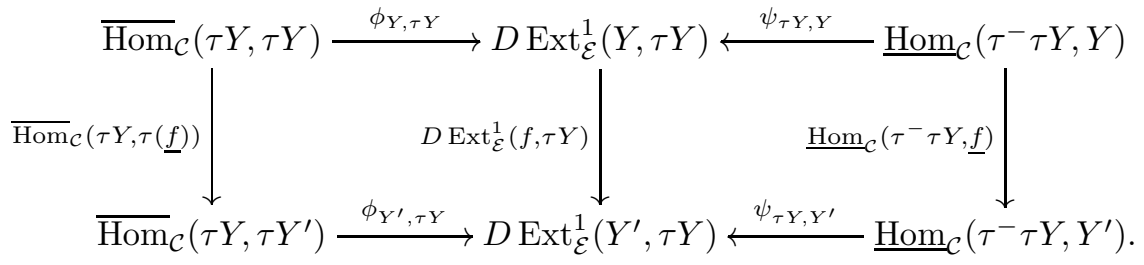

The left square commutes by the definition of $\tau(\underline{f})$, and the right square commutes since the isomorphism $\psi_{\tau Y}$ is natural. By a diagram chasing, we obtain

$$
\tau(\underline{f})=\left(\phi_{Y^{\prime}, \tau Y}^{-1} \circ \psi_{\tau Y, Y^{\prime}}\right)\left(\underline{f} \circ \underline{\epsilon_{Y}}\right) .
$$

We have the following commutative diagram

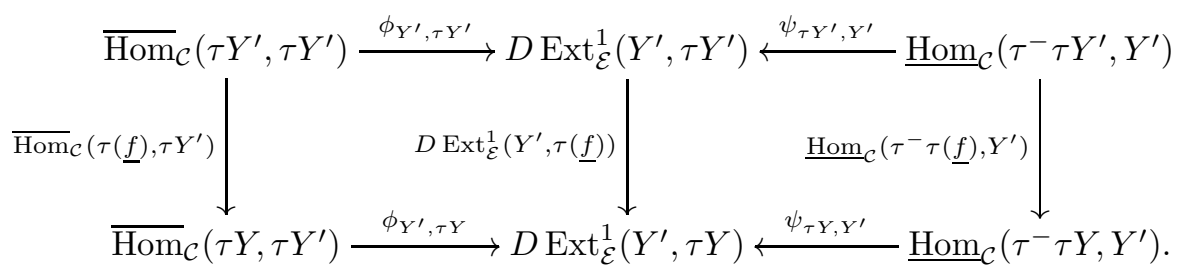

The right square commutes by the definition of $\tau^{-} \tau(\underline{f})$. By a diagram chasing, we obtain

$$
\tau(\underline{f})=\left(\phi_{Y^{\prime}, \tau Y}^{-1} \circ \psi_{\tau Y, Y^{\prime}}\right)\left(\underline{\epsilon}_{Y^{\prime}} \circ \tau^{-} \tau(\underline{f})\right) .
$$

We then obtain $\underline{f} \circ \underline{\epsilon_{Y}}=\underline{\epsilon_{Y^{\prime}}} \circ \tau^{-} \tau(\underline{f})$. It follows that $\underline{\epsilon}$ is a natural transformation.

(2) The proof is similar.

The following result strengthens [8, Proposition 3.3].

Proposition 3.4. The natural transformations $\underline{\epsilon}$ and $\bar{\eta}$ are both isomorphisms. Hence, the functors $\tau$ and $\tau^{-}$are quasi-inverse to each other.

Proof. We only prove that $\underline{\epsilon_{Y}}$ is an isomorphism for each $Y \in \mathcal{C}_{r}$. We may assume that $Y$ is indecomposable and non-projective in $\mathcal{C}$. Let $\alpha=\psi_{\tau Y, \tau^{-} \tau Y}\left(\underline{\operatorname{Id}_{\tau^{-} \tau Y}}\right)$ in $D \operatorname{Ext}_{\mathcal{E}}^{1}\left(\tau^{-} \tau Y, \tau Y\right)$ and let $\beta=\phi_{Y, \tau Y}\left(\overline{\operatorname{Id}_{\tau Y}}\right)$ in $D \operatorname{Ext}_{\mathcal{E}}^{1}(Y, \tau Y)$. By the definition of $\underline{\epsilon_{Y}}$, we have $\beta=\psi_{\tau Y, Y}\left(\underline{\epsilon_{Y}}\right)$.

Consider the following commutative diagram

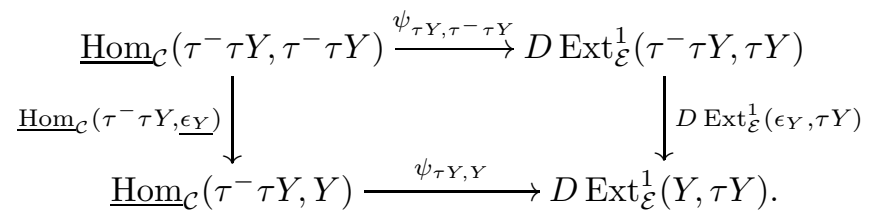

By a diagram chasing, we obtain

$$
\begin{aligned}
\beta & =\psi_{\tau Y, Y}\left(\underline{\epsilon_{Y}}\right) \\
& =\left(\psi_{\tau Y, Y} \circ \underline{\operatorname{Hom}}_{\mathcal{C}}\left(\tau^{-} \tau Y, \underline{\epsilon_{Y}}\right)\right)\left(\underline{\operatorname{Id}_{\tau^{-} \tau Y}}\right) \\
& \left.=\left(D \operatorname{Ext}_{\mathcal{E}}^{1}\left(\epsilon_{Y}, \tau Y\right) \circ \psi_{\tau Y, \tau^{-} \tau Y}\right) \underline{\left(\mathrm{Id}_{\tau^{-} \tau Y}\right.}\right) \\
& =D \operatorname{Ext}_{\mathcal{E}}^{1}\left(\epsilon_{Y}, \tau Y\right)(\alpha) \\
& =\alpha \circ \operatorname{Ext}_{\mathcal{E}}^{1}\left(\epsilon_{Y}, \tau Y\right) .
\end{aligned}
$$


Here, the third equality holds by the commutative diagram. The fourth equality holds by the definition of $\alpha$.

By Lemma 2.2(1), there exists an almost split conflation $\delta: X \rightarrow E \rightarrow Y$. By Lemma 2.1(1), we have a natural isomorphism $\phi^{\prime}: \overline{\operatorname{Hom}}_{\mathcal{C}}(-, X) \rightarrow D \operatorname{Ext}_{\mathcal{E}}^{1}(Y,-)$ such that $\phi_{X}^{\prime}\left(\overline{\operatorname{Id}_{X}}\right)(\delta) \neq 0$. Setting $\beta^{\prime}=\phi_{X}^{\prime}\left(\overline{\operatorname{Id}_{X}}\right)$, we have $\beta^{\prime}(\delta) \neq 0$. By Yoneda's lemma, there exists some $s: X \rightarrow \tau Y$ such that $\overline{\operatorname{Hom}}_{\mathcal{C}}(-, \bar{s})=\phi_{Y}^{-1} \circ \phi^{\prime}$. We obtain

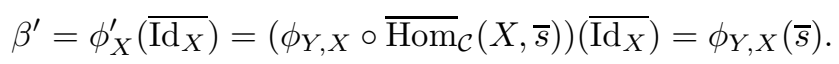

Consider the following commutative diagram

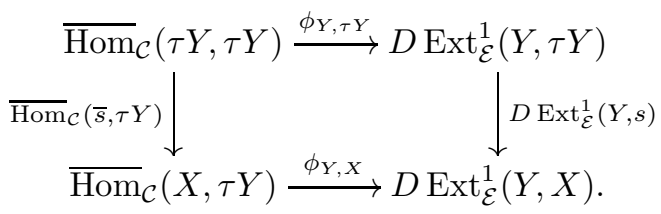

By a diagram chasing, we obtain

$$
\begin{aligned}
\beta^{\prime} & =\phi_{Y, X}(\bar{s}) \\
& =\left(\phi_{Y, X} \circ \overline{\operatorname{Hom}}_{\mathcal{C}}(\bar{s}, \tau Y)\right)\left(\overline{\operatorname{Id}_{\tau Y}}\right) \\
& =\left(D \operatorname{Ext}_{\mathcal{E}}^{1}(Y, s) \circ \phi_{Y, \tau Y}\right)\left(\overline{\operatorname{Id}_{\tau Y}}\right) \\
& =D \operatorname{Ext}_{\mathcal{E}}^{1}(Y, s)(\beta) \\
& =\beta \circ \operatorname{Ext}_{\mathcal{E}}^{1}(Y, s) .
\end{aligned}
$$

Here, the third equality holds by the commutative diagram. The fourth equality holds by the definition of $\beta$. Since $\beta=\alpha \circ \operatorname{Ext}_{\mathcal{E}}^{1}\left(\epsilon_{Y}, \tau Y\right)$, we obtain

$$
\beta^{\prime}=\alpha \circ \operatorname{Ext}_{\mathcal{E}}^{1}\left(\epsilon_{Y}, \tau Y\right) \circ \operatorname{Ext}_{\mathcal{E}}^{1}(Y, s) .
$$

Then we have that

$$
0 \neq \beta^{\prime}(\delta)=\alpha\left((s . \delta) . \epsilon_{Y}\right)=\alpha\left(s .\left(\delta . \epsilon_{Y}\right)\right) .
$$

In particular, the conflation $\delta . \epsilon_{Y}$ is non-split. It follows that $\epsilon_{Y}: \tau^{-} \tau Y \rightarrow Y$ is a retraction in $\mathcal{C}$, since $\delta$ is almost split. By Lemma 3.2(1), we have that $\tau^{-} \tau Y \simeq Y$ in $\underline{\mathcal{C}}$. It follows that $\underline{\epsilon_{Y}}$ is an isomorphism in $\underline{\mathcal{C}}$.

The following lemma shows that $\left(\tau^{-}, \tau\right)$ forms an adjoint pair, with unit $\bar{\eta}$ and counit $\underline{\epsilon}$; see [10, Section IV.1].

Lemma 3.5. We have $\tau\left(\underline{\epsilon_{Y}}\right) \circ \overline{\eta_{\tau Y}}=\overline{\operatorname{Id}_{\tau Y}}$ for each $Y \in \underline{\mathcal{C}_{r}}$, and $\underline{\epsilon_{\tau^{-} X}} \circ \tau^{-}\left(\overline{\eta_{X}}\right)=$ $\underline{\mathrm{Id}_{\tau-X}}$ for each $X \in \overline{\mathcal{C}_{l}}$.

Proof. We only prove the first equality. We have the following commutative diagram

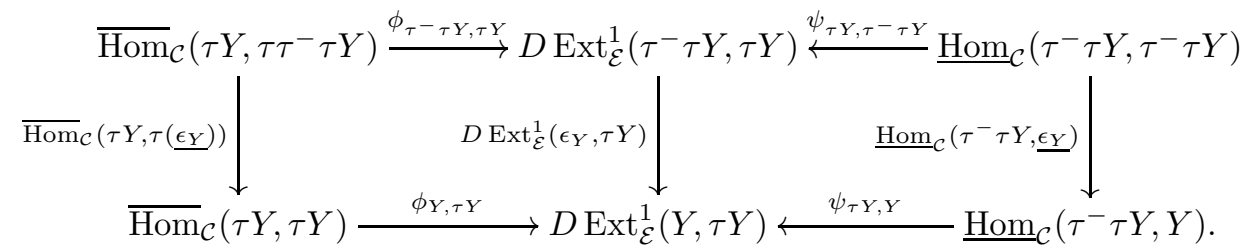


The left square commutes by the definition of $\tau\left(\underline{\epsilon_{Y}}\right)$, and the right square commutes since $\psi_{\tau Y}$ is natural. By a diagram chasing, we obtain

$$
\begin{aligned}
\overline{\overline{\operatorname{Id}}_{\tau Y}} & =\left(\phi_{Y, \tau Y}^{-1} \circ \psi_{\tau Y, Y}\right)\left(\underline{\epsilon_{Y}}\right) \\
& =\left(\phi_{Y, \tau Y}^{-1} \circ \psi_{\tau Y, Y} \circ \underline{\operatorname{Hom}}_{\mathcal{C}}\left(\tau^{-} \tau Y, \underline{\epsilon_{Y}}\right)\right)\left(\underline{\mathrm{Id}_{\tau^{-} \tau Y}}\right) \\
& =\left(\overline{\operatorname{Hom}}_{\mathcal{C}}\left(\tau Y, \tau\left(\underline{\epsilon_{Y}}\right)\right) \circ \phi_{Y, \tau Y}^{-1} \circ \psi_{\tau Y, Y}\right)\left(\underline{\operatorname{Id}_{\tau^{-} \tau Y}}\right) \\
& =\overline{\operatorname{Hom}}_{\mathcal{C}}\left(\tau Y, \tau\left(\underline{\epsilon_{Y}}\right)\right)\left(\overline{\eta_{\tau Y}}\right) \\
& =\tau\left(\underline{\epsilon_{Y}}\right) \circ \bar{\eta}_{\tau Y} .
\end{aligned}
$$

Here, the first equality holds by the definition of $\epsilon_{Y}$. The third equality holds by the commutative diagram. The fourth equality holds by the definition of $\overline{\eta_{\tau Y}}$.

Definition 3.6. Let $\mathcal{C}$ be a Hom-finite Krull-Schmidt exact category. We call the sextuple obtained above

$$
\left\{\mathcal{C}_{r}, \mathcal{C}_{l}, \phi, \psi, \tau, \tau^{-}\right\}
$$

the generalized Auslander-Reiten duality on $\mathcal{C}$ and call the functors $\tau$ and $\tau^{-}$the generalized Auslander-Reiten translation functors.

We mention that the functor $\tau$ depends on $\phi$ and the functor $\tau^{-}$depends on $\psi$. Then $\mathcal{C}$ has Auslander-Reiten duality in the sense of [8] if and only if $\mathcal{C}_{l}=\mathcal{C}=\mathcal{C}_{r}$.

Remark 3.7. Let $(\mathcal{C}, \mathcal{E})$ be a Frobenius category. Then the projectively stable category and the injectively stable category of $\mathcal{C}$ are the same and have a natural triangulated structure. The generalized Auslander-Reiten duality on $\mathcal{C}$ gives the generalized Serre duality on $\underline{\mathcal{C}}$ in the sense of [5]. More precisely, we have $(\underline{\mathcal{C}})_{r}=\underline{\mathcal{C}_{r}}$ and $(\underline{\mathcal{C}})_{l}=\overline{\mathcal{C}_{l}}$. Let $\Sigma$ be the translation functor of $\underline{\mathcal{C}}$. Then the functor $\Sigma \tau: \underline{\mathcal{C}_{r}} \rightarrow \overline{\overline{\mathcal{C}_{l}}}$ gives the generalized Serre functor of $\underline{\mathcal{C}}$.

\section{The CATEGory OF FINITELY PRESENTED REPRESENTATIONS}

From now on, we let $k$ be a field and $Q=\left(Q_{0}, Q_{1}\right)$ be a quiver. Here, $Q_{0}$ is the set of vertices and $Q_{1}$ is the set of arrows. Given an arrow $\alpha: a \rightarrow b$, denote by $s(\alpha)=a$ its source and by $t(\alpha)=b$ its target. A path $p$ of length $l \geq 1$ is a sequence of arrows $\alpha_{l} \cdots \alpha_{2} \alpha_{1}$ such that $s\left(\alpha_{i+1}\right)=t\left(\alpha_{i}\right)$ for each $i=1,2, \ldots, l-1$. We let $s(p)=s\left(\alpha_{1}\right)$ and $t(p)=t\left(\alpha_{l}\right)$. A left infinite path is an infinite sequence of arrows $\alpha_{1} \alpha_{2} \cdots \alpha_{n} \cdots$ such that $s\left(\alpha_{i}\right)=t\left(\alpha_{i+1}\right)$ for each $i \geq 1$. Dually, a right infinite path is an infinite sequence of arrows $\cdots \alpha_{n} \cdots \alpha_{2} \alpha_{1}$ such that $s\left(\alpha_{i+1}\right)=t\left(\alpha_{i}\right)$ for each $i \geq 1$.

Recall that a quiver $Q$ is locally finite if for each $a \in Q_{0}$, the set of arrows starting at $a$ or ending at $a$ is finite. A quiver $Q$ is interval-finite if for any $a, b \in Q_{0}$, the set of paths $p$ with $s(p)=a$ and $t(p)=b$ is finite. We will assume that $Q$ is locally finite and interval-finite.

A representation $M$ of $Q$ is called locally finite dimensional if $M(a)$ is finite dimensional for each $a \in Q_{0}$, and is finite dimensional if moreover $\bigoplus_{a \in Q_{0}} M(a)$ is finite dimensional. Denote by $\operatorname{rep}(Q)$ the category of locally finite dimensional representations, and by $\operatorname{rep}^{b}(Q)$ the full subcategory formed by finite dimensional representations. Then the Matlis duality induces a duality $D: \operatorname{rep}(Q) \rightarrow \operatorname{rep}\left(Q^{\mathrm{op}}\right)$, which sends $\operatorname{rep}^{b}(Q)$ into $\operatorname{rep}^{b}\left(Q^{\mathrm{op}}\right)$. Here, $Q^{\mathrm{op}}$ is the opposite quiver of $Q$.

Recall from [4, Section 2] that the path-category $\mathcal{Q}$ of $Q$ has $Q_{0}$ as the set of objects; if $x, y \in Q_{0}$, the morphisms from $x$ to $y$ are the linear combinations of paths 
from $x$ to $y$. It is well known that $\operatorname{rep}(Q)$ is equivalent to the category of covariant functors from $\mathcal{Q}$ to $k$-mod. We denote by $\operatorname{proj}(Q)$ the full subcategory of $\operatorname{rep}(Q)$ formed by objects which corresponds to the finite direct sums of representable functors $\operatorname{Hom}_{\mathcal{Q}}(x,-)$ for some objects $x \in \mathcal{Q}$.

A representation $M$ is called finitely presented, if there exists an exact sequence $P_{1} \stackrel{u}{\rightarrow} P_{0} \stackrel{v}{\rightarrow} M \rightarrow 0$ with $P_{1}, P_{0} \in \operatorname{proj}(Q)$. The exact sequence is called a presentation of $M$; it is a minimal presentation if moreover the kernels of $u$ and $v$ are superfluous in $P_{1}$ and $P_{0}$, respectively. Denote by $\operatorname{rep}^{+}(Q)$ the full subcategory of $\operatorname{rep}(Q)$ formed by the finitely presented representations. We mention that $\operatorname{rep}^{+}(Q)$ is a Hom-finite hereditary abelian subcategory, which is closed under extensions in $\operatorname{rep}(Q)$; moreover, we have $\operatorname{rep}^{b}(Q) \subseteq \operatorname{rep}^{+}(Q)$; see [3, Proposition 1.15].

Denote by $A=k Q$ the path algebra of $Q$. Then $A$ admits a complete set of primitive orthogonal idempotents $\left\{e_{a} \mid a \in Q_{0}\right\}$. Recall that a left $A$-module $M$ is called unitary if $A M=M$, which is equivalent to $M=\bigoplus_{a \in Q_{0}} e_{a} M$. Then ${ }_{A} A$ is a left unitary $A$-modules. It is well known that the category of representations of $Q$ is equivalent to the category of unitary left $A$-modules. For convenience, we will identify representations of $Q$ with the corresponding unitary left $A$-modules. We mention that the contravariant functor $\operatorname{Hom}_{A}(-, A): \operatorname{proj}(Q) \rightarrow \operatorname{proj}\left(Q^{\mathrm{op}}\right)$ is a duality; see [3, Lemma 1.18].

Let $P_{1} \stackrel{g}{\rightarrow} P_{0} \rightarrow M \rightarrow 0$ be a minimal presentation of a representation $M$ in $\operatorname{rep}(Q)$. The cokernel of $\operatorname{Hom}_{A}(g, A)$ is called the transpose $\operatorname{Tr} M$ of $M$. Here, $\operatorname{Tr} M$ has no nonzero projective direct summands. We observe that a morphism $f: X \rightarrow Y$ in $\operatorname{rep}^{+}(Q)$ is projectively trivial if and only if it factors through a projective object, since $\operatorname{rep}^{+}(Q)$ has enough projectives. Then we obtain a duality $\operatorname{Tr}: \operatorname{rep}^{+}(Q) \rightarrow \operatorname{rep}^{+}\left(Q^{\text {op }}\right)$. Here, $\operatorname{rep}^{+}(Q)$ can be embedded in $\operatorname{rep}^{+}(Q)$ as a full subcategory, since rep ${ }^{+}(Q)$ is hereditary. Then we have a contravariant functor

$$
\operatorname{Tr}: \operatorname{rep}^{+}(Q) \longrightarrow \operatorname{rep}^{+}\left(Q^{\mathrm{op}}\right) \text {. }
$$

The following lemma is contained in the proof of [3, Theorem 2.8].

Lemma 4.1. Let $L, M \in \operatorname{rep}(Q)$.

(1) If $M$ lies in $\mathrm{rep}^{+}(Q)$, then there exists an isomorphism $\operatorname{Hom}(L, D \operatorname{Tr} M) \simeq$ $D \operatorname{Ext}^{1}(M, L)$, which is natural in $L$ and $M$.

(2) If $M$ lies in $\operatorname{rep}^{b}(Q)$, then there exists an isomorphism $\operatorname{Hom}(\operatorname{Tr} D M, L) \simeq$ $D \operatorname{Ext}^{1}(L, M)$, which is natural in $L$ and $M$.

The following lemma is due to [3, Theorem 2.8, Corollary 2.9 and Propositions 3.6].

Lemma 4.2. Let $M \in \operatorname{rep}^{+}(Q)$ be an indecomposable representation.

(1) If $M$ is non-projective, then there exists an almost split sequence $0 \rightarrow$ $D \operatorname{Tr} M \rightarrow E \rightarrow M \rightarrow 0$ in $\operatorname{rep}(Q)$.

(2) If $M$ is non-injective and lies in $\operatorname{rep}^{b}(Q)$, then there exists an almost split sequence $0 \rightarrow M \rightarrow E \rightarrow \operatorname{Tr} D M \rightarrow 0$ in $\mathrm{rep}^{+}(Q)$.

(3) Assume that $0 \rightarrow M \rightarrow E \rightarrow N \rightarrow 0$ is an exact sequence in $\operatorname{rep}(Q)$. Then it is an almost split sequence in $\operatorname{rep}^{+}(Q)$ if and only if it is an almost split sequence in $\operatorname{rep}(Q)$ with $M \in \operatorname{rep}^{b}(Q)$.

Lemma 4.3. Let $f: X \rightarrow Y$ be an injectively trivial morphism in $\operatorname{rep}^{+}(Q)$. If $Y$ has no nonzero injective direct summands and lies in $\operatorname{rep}^{b}(Q)$, then we have $f=0$. 
Proof. We may assume that $Y$ is indecomposable. By Lemma 4.2(2), we have an almost split sequence $0 \rightarrow Y \rightarrow E \rightarrow \operatorname{Tr} D Y \rightarrow 0$ in $\operatorname{rep}^{+}(Q)$. By Lemma 4.1(1), we have $\operatorname{Hom}(X, D \operatorname{Tr} \operatorname{Tr} D Y) \simeq D \operatorname{Ext}^{1}(\operatorname{Tr} D Y, X)$. By Lemmas 2.1(1), we have $\overline{\operatorname{Hom}}(X, Y) \simeq D \operatorname{Ext}^{1}(\operatorname{Tr} D Y, X)$. Observe that $D \operatorname{Tr} \operatorname{Tr} D Y \simeq Y$. Then we have $\overline{\operatorname{Hom}}(X, Y) \simeq \operatorname{Hom}(X, Y)$. Then the result follows.

Now, we can describe the generalized Auslander-Reiten duality on $\operatorname{rep}^{+}(Q)$. Given a collection $\mathcal{S}$ of objects, denote by add $\mathcal{S}$ the category of direct summands of finite direct sums of objects in $\mathcal{S}$.

Proposition 4.4. Let $Q$ be a locally finite interval-finite quiver. Set $\mathcal{C}=\operatorname{rep}^{+}(Q)$. Then we have

$$
\mathcal{C}_{r}=\left\{X \in \mathcal{C} \mid \operatorname{Tr} X \in \operatorname{rep}^{b}\left(Q^{\mathrm{op}}\right)\right\}
$$

and

$$
\mathcal{C}_{l}=\operatorname{add}\left\{X \mid X \in \operatorname{rep}^{b}(Q) \text { or } X \text { is an injective object in } \mathcal{C}\right\} .
$$

Moreover, the functors $D \operatorname{Tr}$ and $\operatorname{Tr} D$ induce the generalized Auslander-Reiten translation functors.

Denote by $\left(\mathcal{C}_{r}\right)_{\mathcal{P}}$ the full subcategory of $\mathcal{C}_{r}$ formed by objects without nonzero projective direct summands, and by $\left(\mathcal{C}_{l}\right)_{\mathcal{I}}$ the full subcategory of $\mathcal{C}_{l}$ formed by objects without nonzero injective direct summands. We obtain the induced functors $D \operatorname{Tr}:\left(\mathcal{C}_{r}\right)_{\mathcal{P}} \rightarrow\left(\mathcal{C}_{l}\right)_{\mathcal{I}}$ and $\operatorname{Tr} D:\left(\mathcal{C}_{l}\right)_{\mathcal{I}} \rightarrow\left(\mathcal{C}_{r}\right)_{\mathcal{P}}$. Since $\mathcal{C}$ is hereditary and has enough projectives, the canonical functor $\left(\mathcal{C}_{r}\right)_{\mathcal{P}} \rightarrow \underline{\mathcal{C}_{r}}$ is an equivalence. By Lemma 4.3, the canonical functor $\left(\mathcal{C}_{l}\right)_{\mathcal{I}} \rightarrow \overline{\mathcal{C}_{l}}$ is an equivalence. Then we have the induced functors $D \operatorname{Tr}: \underline{\mathcal{C}_{r}} \rightarrow \overline{\mathcal{C}_{l}}$ and $\operatorname{Tr} D: \overline{\mathcal{C}_{l}} \rightarrow \underline{\mathcal{C}_{r}}$.

Proof. We observe that $\operatorname{Tr} P=0$ for each projective object $P$. Then the first equality follows from Proposition 2.4(1) and Lemma 4.2(1) and (3). The second equality follows from Proposition 2.4(2) and Lemma 4.2(2) and (3).

Let $X \in \mathcal{C}$. By Lemmas 4.1(1) and 4.3, for each $Y \in\left(\mathcal{C}_{r}\right)_{\mathcal{P}}$, we have an isomorphism $\overline{\operatorname{Hom}}(X, D \operatorname{Tr} Y) \simeq D \operatorname{Ext}^{1}(Y, X)$, which is natural in $X$ and $Y$ since $D \operatorname{Tr} Y$ has no nonzero injective direct summands. Similarly, for each $Y \in\left(\mathcal{C}_{l}\right)_{\mathcal{I}}$, we have $\underline{\operatorname{Hom}}(\operatorname{Tr} D Y, X) \simeq D \operatorname{Ext}^{1}(X, Y)$, which is natural in $X$ and $Y$. Then the result follows from the construction of generalized Auslander-Reiten translation functors.

Combining Proposition 4.4 and [3, Theorem 3.7], we have the following direct consequence.

Corollary 4.5. Let $Q$ be a connected locally finite interval-finite quiver. Then the category $\operatorname{rep}^{+}(Q)$ has Auslander-Reiten duality if and only if $Q$ has neither left infinite path nor right infinite path, or else $Q$ is of the form

$$
\cdots \longrightarrow 0 \longrightarrow \cdots \longrightarrow 0 \longrightarrow 0 \text {. }
$$

\section{ACKNOWLEDGEMENTS}

The author thanks his advisor Professor Xiao-Wu Chen for his guidance and encouragement, and thanks Dawei Shen for some suggestions and comments. 


\section{REFERENCES}

[1] M. Auslander, Functors and morphisms determined by objects, in Representation theory of algebras (Proc. Conf., Temple Univ., Philadelphia, Pa., 1976), vol. 37 of Lecture Notes in Pure Appl. Math., Dekker, New York, 1978, 1-244.

[2] M. Auslander, I. Reiten, and S. O. Smalø, Representation theory of Artin algebras, vol. 36 of Cambridge Studies in Advanced Mathematics, Cambridge Univ. Press, Cambridge, 1995.

[3] R. Bautista, S. Liu, and C. Paquette, Representation theory of strongly locally finite quivers, Proc. Lond. Math. Soc. (3) 106 (2013), 97-162.

[4] K. Bongartz and P. Gabriel, Covering spaces in representation-theory, Invent. Math. 65 (1982), 331-378.

[5] X.-W. Chen, Generalized Serre duality, J. Algebra 328 (2011), 268-286.

[6] P. Gabriel and A. V. Roiter, Representations of finite-dimensional algebras, in Algebra VIII, vol. 73 of Encyclopaedia Math. Sci., Springer, Berlin, 1992, 1-177.

[7] B. Keller, Chain complexes and stable categories, Manuscripta Math. 67 (1990), 379-417.

[8] H. Lenzing and R. Zuazua, Auslander-Reiten duality for abelian categories, Bol. Soc. Mat. Mexicana (3) 10 (2004), 169-177.

[9] S. Liu, P. Ng, and C. Paquette, Almost split sequences and approximations, Algebr. Represent. Theory 16 (2013), 1809-1827.

[10] S. Mac Lane, Categories for the working mathematician, vol. 5 of Graduate Texts in Mathematics, Springer-Verlag, New York-Berlin, 1971.

[11] D. Quillen, Higher algebraic $K$-theory: $I$, in Algebraic $K$-theory I: Higher $K$-theories (Proc. Conf., Battelle Memorial Inst., Seattle, Wash., 1972), vol. 341 of Lecture Notes in Math., Springer, Berlin, 1973, 85-147.

School of Mathematical Sciences, University of Science and Technology of China, Hefei 230026, PR CHINA

E-mail address: jiaopjie@mail.ustc.edu.cn 